\title{
ECOLOGICAL STUDIES OF VEGETATION IN SHORT-TAILED SHEARWATER COLONIES IN TASMANIA
}

\author{
by M.J. Brown, N. Maruyama and K.J. Williams
}

(with two text-figures and three tables)

Brown, M.J., Maruyama, N. \& Williams, K.J., 1993 (31:viii): Ecological studies of vegetation in short-tailed shearwater colonies in Tasmania. Pap. Proc. R. Soc. Tasm. 127: 11-16. https://doi.org/10.26749/rstpp.127.11

Forestry Commission, 199 Macquarie Street, Hobart, Tasmania, Australia 7000 (MJB and KJW); University Tokyo Noko, Tokyo, Japan (NM).

Extensive modification of coastal vegetation at the Pineapples and Cape Deslacs rookeries is related to the burrowing, trampling and guano deposition of short-tailed shearwaters (Puffinus tenuirostris). Ordination and classification of the associated vegetation and measurement of soil nutrient levels demonstrate two distinct but interacting phases of vegetation succession. These are affected by coastal influence, edaphic factors and the presence or absence of nesting areas.

Key Words: vegetation, soil nutrients, shearwater, ecological studies, Tasmania.

\section{INTRODUCTION}

The short-tailed shearwater (Puffinus tenuirostris) is a major ecological, cultural and sometimes economic feature of the Tasmanian coastal landscape.

In a classic series of papers, Gillham (1958, 1960, 1961, 1965) elucidated the characteristics of the vegetation of Tasmanian short-tailed shearwater colonies in the Furneaux Group of islands in Bass Strait. Her papers also provide some indications of the soil conditions which prevail at rookery sites.

During the 1970s and 1980s staff from the State Wildlife agency (currently the Parks and Wildlife Service, Department of Environment and Land Management) have continued the long-term studies, begun in the late 1950 s by $D$. Serventy and then carried on by V. Serventy, into the life cycle and dynamics of the short-tailed shearwater (e.g. Serventy 1958, 1967, 1968, Skira et al. 1986). These studies have been broadened to encompass statewide inventory and monitoring of the effects of commercial and non-commercial exploitation of the birds for human consumption (e.g. Naarding 1981, Skira 1990).

The succession of vegetation in coastal areas of Tasmania has been examined in detail at several sites around the state. Thus Bowden \& Kirkpatrick (1974) have outlined the progression of vegetation from the shore to the saltmarsh hinterland at Rheban Spit in the southeast and Brown (1980) outlined a putative successional scheme for the western coastal vegetation at Mt Cameron West. More recently Chladil \& Kirkpatrick (1989) undertook a quantitative study of the dune vegetation at Asbestos Range National Park in the north.

Whilst there has been considerable study of the birds and of their habitats, there has been little attempt to relate the dynamics of the birds in situ and the ecological succession of vegetation at the rookeries. The present paper attempts to provide a first examination of these interrelationships at two rookeries, Pineapples and Cape Deslacs, and includes relevant comment from elsewhere.

\section{METHODS}

\section{Studies of Site Vegetation and Soil}

The vegetation at Pineapples and Cape Deslacs (fig. 1) was sampled on several occasions during Dec/Jan 1987/88 and in $1989 / 90$ using $2 \times 2$ m quadrats. Each species was scored for its abundance using a modified Braun-Blanquet score. Vascular plants only were scored.The species nomenclature used in this paper follows Buchanan et al. (1989). The species presence data from Pineapples were subjected to ordination and classification using detrended correspondence analysis (DCA) and TWINSPAN respectively (Hill 1979a, b). Only two axes were selected from the ordination, as the later axes are known to be non-linear combinations of axes one and two (P. Minchin, pers. comm.).

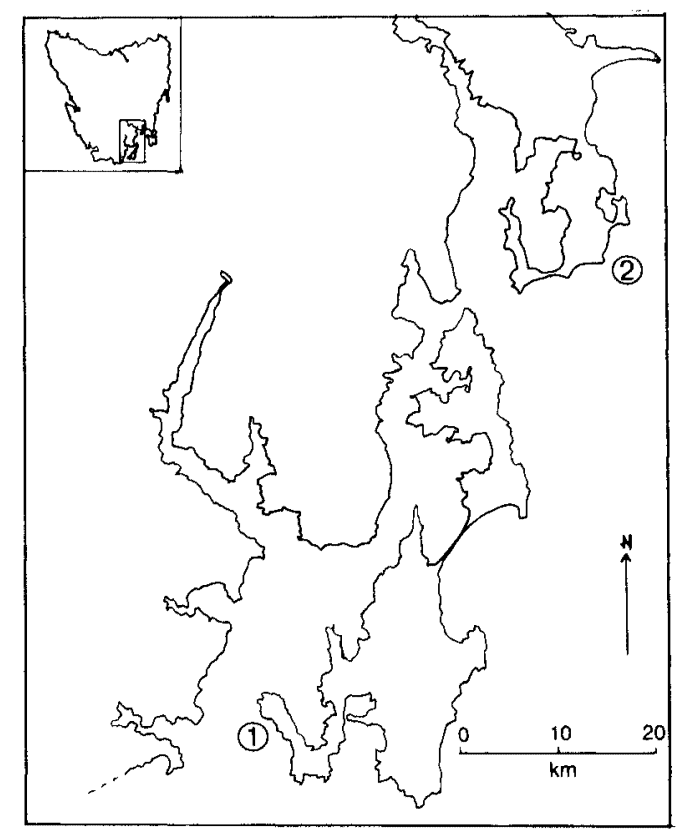

FIG. 1 - Locations of study sites: (1) Pineapples; (2) Cape Deslacs. 
Total vegetation cover, rock cover and sand cover was classified, and an assessment was made of the number of burrows, the recency of occupancy and the amount of disturbance at each site.

Soils were collected at Pineapples (14 samples) and at Cape Deslacs (17 samples) to encompass the range of vegetated environments encountered at each site. Soil analyses were conducted for us by the Tasmanian Department of Primary Industry's analytical laboratories. Soil characteristics measured were $\mathrm{pH}$ (aqueous extract), conductivity (aqueous extract), P, K, N, Ca, Mg and $\mathrm{Na}$ (bicarbonate extract). Organic carbon content was requested but measurement facilities were not available.

In order to analyse numerically the relationships between soils and the vegetation, the data from Pineapples were subjected to factor analysis and subsequent varimax rotation. The DCA scores were incorporated into these analyses together with $\mathrm{pH}$, conductivity and the logarithms of the concentrations of $\mathrm{P}, \mathrm{K}, \mathrm{N}, \mathrm{Ca}, \mathrm{Mg}$, and $\mathrm{Na}$ in ppm.

\section{Site Descriptions}

The rookeries at Pineapples are underlain by aeolian sands which, in turn, overlie Jurassic dolerite. The rookeries are on an exposed westerly aspect and extend from a few metres inland of the unvegetated coastal rocks to about $300 \mathrm{~m}$ inland. The area was burnt in the bushfires of $1966 / 67$, and parts of the area were burnt again in 1982.

The cliffs at Cape Deslacs are formed of Permian sediments (Parmeener supergroup) and rise to $54 \mathrm{~m}$. These cliffs are also overlain by acolian sands, which are of sufficient depth to support short-tailed shearwater colonies. The colonies here are found on all aspects, the bluff forming an indented headland which projects into the sea to the southeast and which also falls away from a high point towards the north. The rookeries occupy the cliff tops and extend about 150 $200 \mathrm{~m}$ inland. This area was probably also burnt in 1967 , but no clear evidence of the age of the vegetation was obtained.

\section{RESULTS}

The results of the analysis by Twinspan are shown in table 1 . The descriptions of the communities at Cape Deslacs and at Pineapples which follow are based on this classification but have been modified to accord with field observations.

\section{The Vegetation}

The plant communities at both sites are of similar composition. Near the coast there is a halophytic community dominated by various combinations of Disphyma australe, Tetragonia implexicoma, Carpobrotus rossii, Stipa stipoides, Leucopogon parviflorus, Poa poiformis, Poa labillardierii, Scirpus nodosus and Rhagodia baccata. These communities have a range of structures depending on their physiographic situation. On deep soils on cliff ledges and declivities, the shrubs predominate and form open shrublands, whilst open grasslands of Stipa stipoides/Poa poiformis or succulent herbfields occur on the shallow soils of the exposed cliff tops and rock faces. Other cliff top shrub elements include Helichrysum reticulatum and Olearia phlogopappa.

Small saline soaks and wet patches are found on drainage lines in shallow soils of the cliffs and support non-succulent salt-tolerant species such as Juncus kraussii, Lobelia alata, Gahnia filum and Schoenus nitens. Where there are no burrows, the vegetation consists of dry or wet heaths dominated by Banksia marginata together with Epacridaceae such as Epacris impressa, E. Lanuginosa and Sprengelia incarnata, Fabaceae (Aotus ericoides, Pultenaea spp., Acacia spp.) and Myrtaceae (Leptospermum scoparium, L. glaucescens, $L$. lanigerum, Melaleuca squarrosa, $M$. gibbosa and $M$. squamea). The heaths are the most species-rich communities at both locations. The vegetation grades through scrubs of Leptospermum spp. and Melaleuca spp. of low diversity to heathy woodlands dominated by Eucalyptus viminalis or $E$. obliqua. The structural and floristic variation in the vegetation is strongly conditioned by exposure, soil depth, fire and drainage.

Thus the halophytic communities develop on the most exposed coastal onshore areas, with the heaths occupying the deeper aeolian sands. Melaleuca and/or Leptospermum scrubs develop along drainage lines or small creeks, and the Eucalyptus woodlands are found on more sheltered sires on deeper soils. Frequent fires promote the heath vegetation at the expense of the scrubs. In the prolonged absence of fire, much of the heath containing the dominant Leptospermum or Melaleuca species would succeed to scrub, with a corresponding loss of species richness. On the other hand, too frequent fire promotes bracken. In the woodlands, infrequent fires result in a heathy or shrubby understorey. Very frequent, low-intensity fires result in bracken understoreys where the soils are sandy but tend to grassy understoreys where the overlying aeolian sands are shallow or where there has been admixing of the nutrient rich dolerite sub-soils and the sands.

The shearwater colonies are found on the deeper sands of the hinterland. Three main communities can be recognised: (1) an open communiry of Poa labillardieri/P. poiformis and Lomandra longifolia, with considerable bare areas and a variable cover of introduced annual species such as Brornus diandrus, Agropyron repens, Cirsium vulgare, Hordeum leporinum and Holcus lanatus;

(2) a dense community dominated by Pteridium esculentum, with few other species present;

(3) a succulent halophytic herbfield community of variable cover but containing Carpobrotus rossii, Tetragonia implexicoma, and Rhagodia baccata, either in pure stands or in any combination.

These three communities represent different successional stages in the life of the rookery. The main determinants of the community present at any one site are degree of trampling and guano deposition, degree of incorporation of dead grass and other organic matter into the soil by the birds, and the effects of fire. The birds thus provide a strong biogenic control over the traditional successional pathways which are found in coastal areas where the birds are absent (e.g. Jackson 1968, Kirkpatrick 1977, Brown 1980, Brown \& Podger 1982). The ordination by detrended correspondence analysis (DCA) of the Pineapples rookery is shown in figure 2 . The encircled quadrats are the four major groups recognised in the Twinspan classification (see table 1). It can be seen from the ordination that the first axis aligns the sites from those with high DCA scores for the heath plots and no burrows, through intermediate values representing 
TABLE 1

TWINSPAN analysis of Pineapples Rookery vegetation data

\begin{tabular}{|c|c|c|c|c|c|}
\hline Vascular plant species & $\begin{array}{l}\text { Burrow-rich sites, } \\
\text { deep sandy soils }\end{array}$ & $\begin{array}{l}\text { Coastal sites, } \\
\text { shallow soils }\end{array}$ & $\begin{array}{l}\text { Bracken- } \\
\text { rich sites }\end{array}$ & $\begin{array}{l}\text { Heath/scrub sites, } \\
\text { no burrows }\end{array}$ & $\begin{array}{l}\text { Twinspan } \\
\text { divisions (3) }\end{array}$ \\
\hline Acaena novae-zelandiae & -------------------- & $1-11--1$ & $1 \ldots-\ldots$ & $-\ldots \ldots \ldots+$ & 000 \\
\hline Juncus pallidus & $\ldots--1-\ldots \ldots \ldots \ldots-\ldots-\ldots$ & $1-\ldots-11-$ & $--1-\cdots$ & $-\ldots \ldots \ldots$ & 000 \\
\hline Leucopogon australis & $-------------1 \cdots---$ & $\ldots--1-$ & $-\ldots-\ldots$ & $\ldots-\ldots \ldots-$ & 000 \\
\hline Lobelia alata & $-\ldots \ldots \ldots-\ldots \ldots \ldots-\ldots$ & 11111111 & $1 \ldots-\ldots$ & $-\ldots-\ldots-\ldots$ & 000 \\
\hline Agrostis avenacea & 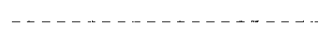 & $1 \ldots \ldots$ & $-\ldots--\ldots$ & $\ldots \ldots \ldots-n$ & 000 \\
\hline Apium prostratum & 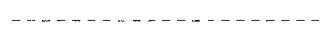 & $1--1-\ldots$ & $\ldots \ldots$ & $\ldots \ldots \ldots$ & 000 \\
\hline Baumea juncea & 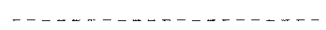 & $11111---$ & $--\ldots \ldots$ & $-\ldots \ldots \ldots-\ldots$ & 000 \\
\hline Epilobium billardierianum & $-\cdots-1-1-1$ & $1 \ldots-\ldots$ & $-\ldots-\ldots$ & $-\ldots-n-\cdots$ & 000 \\
\hline Gahnia filum & $-\ldots-\ldots-\ldots-\ldots-\ldots-------$ & $11-11-\cdots$ & $\ldots-\ldots$ & $-\ldots-\ldots-\cdots$ & 000 \\
\hline Helichrysum reticulatum & $--\ldots+-\ldots,-\ldots--\infty \ldots--$ & $----1---$ & ------ & -------- & 000 \\
\hline Juncus kraussii & 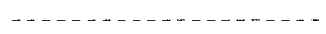 & $1 \ldots \ldots$ & $\ldots \ldots-\ldots$ & $\ldots \ldots \ldots \ldots$ & 000 \\
\hline Oxalis corniculata & 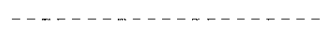 & $1--1---$ & ------ & -------- & 000 \\
\hline Rumex crispus & 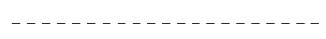 & $1--1--1-$ & $\ldots \ldots$ & $\ldots \ldots-\ldots \ldots$ & 000 \\
\hline Stipa stipoides & 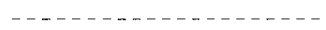 & $----1---$ &.----- & -------- & 000 \\
\hline Veronica gracilis & $-\ldots-\ldots-\ldots-\ldots-\ldots-\ldots-\ldots$ & $1 \ldots \ldots$ & $\ldots \ldots$ & $\ldots \ldots \ldots \ldots$ & 000 \\
\hline Sonchus megalocarpus & $-------1--------1$ & $--i 11--$ & $-\ldots \ldots$ & $---\ldots---$ & 000 \\
\hline Cirsium vulgare & $\ldots \ldots \ldots-\ldots$ & $1 \cdots+\ldots 1-$ & $-\ldots-\ldots$ & $-\ldots \ldots-\ldots-$ & 001 \\
\hline Scirpus nodosus & $----1--11111111--11$ & $111---1-$ & $\ldots-\ldots$ & $--1-\cdots \cdots$ & 001 \\
\hline Bromus diandrus & $---111111-1-1-11--$ & $-\ldots--1$ & $-\ldots---$ & $-\ldots------$ & 001 \\
\hline Carpobrotus rossii & 1-1-111111111111-1--- & $11-1-\cdots$ & ------ & $-\ldots \ldots-\ldots$ & 001 \\
\hline Holcus lanatus & $\ldots-11 \ldots \ldots \ldots$ & $\ldots \ldots$ & $---\cdots-$ & $--\ldots-\ldots-\cdots$ & 001 \\
\hline Hordeum murinum ssp. leporinum & $----11-1--\rightarrow-------$ & $-\ldots-\ldots-$ & $---\ldots$ & $\ldots-\ldots \ldots$ & 001 \\
\hline Solanum laciniatum & $\ldots \ldots \ldots \ldots-1-n-111--$ & $--\ldots-\ldots-$ & ------- & --------- & 001 \\
\hline Rhagodia baccata & $11-1 \ldots \ldots \ldots \ldots$ & $\ldots \ldots$ & $-\ldots \ldots--$ & $\ldots \ldots \ldots-\ldots$ & 001 \\
\hline Tetragonia implexicoma & $11-1----1--11111-----$ & $1-------$ & ------ & $--1-\cdots---$ & 001 \\
\hline Poa labillardieri & $-1--1--111-11-111111$ & 11111111 & $1-1---$ & $-----11--$ & 001 \\
\hline Lomandra longifolia & $111-1-1 \ldots--1111--1$ & $1111--11$ & $1---1-1$ & $11-11111-1$ & 010 \\
\hline Pteridium esculentum & $1-11--1---1-111--111-$ & $---1-111$ & 1111111 & $11111111-1$ & 010 \\
\hline Senecio vulgaris & $1-\ldots-\ldots$ & $\ldots-\ldots-$ & $1 \ldots \ldots$ & $-\ldots-----$ & 010 \\
\hline Dianella tasmanica & $\ldots-11+\ldots \ldots \ldots$ & $--1-111$ & $1---1-$ & $------1--$ & 011 \\
\hline Leucopogon parviflorus & ------------------- & ------1 & $1-----$ & $----\cdots--$ & 011 \\
\hline Goodenia ovata & 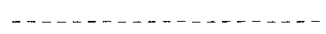 & $\cdots--11-$ & $111---$ & $-\cdots-111--$ & 10 \\
\hline Melaleuca gibbosa & 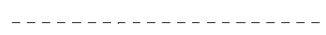 & $---1--1$ & $1-\ldots-\ldots$ & $\ldots-1111 \ldots$ & 10 \\
\hline Melaleuca squarrosa & $-\ldots+\ldots+\ldots,-\ldots+\ldots$ & $-----1--$ & $111---$ & $---1111--$ & 10 \\
\hline Eucalyptus obliqua & 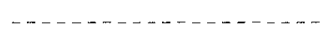 & $\ldots-\ldots-n$ & $-\cdots-1$ & $---1-----$ & 110 \\
\hline Hydrocotyle hirta & 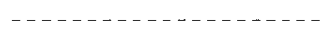 & $-\ldots-\ldots-$ & $1-\ldots--$ & $-\ldots-\ldots-\ldots$ & 110 \\
\hline Leptospermum scoparium & ------------------ & ------1 & $1-1---$ & $--11111 \hat{i} 11$ & 110 \\
\hline Olearia floribunda & $\ldots \ldots \ldots \ldots$ & $\ldots \ldots \ldots$ & $--\cdots-1$ & $-\ldots-11-1$ & 110 \\
\hline Gahnia grandis & - - - - - - - - - - - - - & ------- & $1----$ & $----1111-$ & 1.11 \\
\hline Acacia myrtifolia & 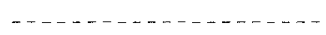 & $-\ldots \ldots \ldots-$ & $--\ldots-\ldots$ & --1111111 & 111 \\
\hline Acacia verticillata & $-\ldots-\ldots, \ldots+\ldots-\ldots,-\ldots$ & $-\ldots-n--$ & $---\ldots$ & $---1-\ldots-\ldots$ & 111 \\
\hline Amperea xiphoclada & --------------------- & -------- & $-\ldots-\ldots$ & $1---1111-1$ & 111 \\
\hline Baumea acuta & $-\ldots-\ldots-\ldots-\ldots-\ldots-\ldots$ &.------ & ------ & $-----1---$ & 111 \\
\hline Allocasuarina monilifera & 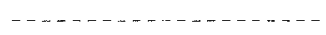 & $\ldots-\ldots \ldots-$ & $--\ldots \ldots-$ & $-11-1111-1$ & 111 \\
\hline Cassytha glabella & $\ldots-\ldots+\ldots-\ldots-\ldots-\ldots$ & $\ldots \ldots-\ldots$ & $---\ldots$ & $---1-\ldots$ & $\hat{1} 11$ \\
\hline Epacris impressa & ------------------- & $--\ldots---$ & $-\ldots---$ & $---1111-1$ & 111 \\
\hline Gonocarpus tetragynus & $\ldots \ldots \ldots \ldots$ & $\ldots-\ldots-$ & $--\ldots+\cdots$ & $\ldots-11--1$ & 111 \\
\hline Lepidosperma laterale & 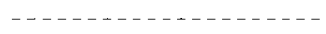 & $------1-$ & ------ & $1--111111-$ & 111 \\
\hline Leptocarpus tenax & - n $\ldots \ldots \ldots$ & $\ldots \ldots \ldots$ & $-\ldots \ldots-$ & $-\ldots-111--$ & 111 \\
\hline Olearia phlogopappa & 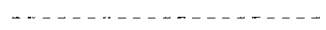 & $-\ldots-\ldots-$ & $---\ldots$ & $1-\ldots \ldots$ & 111 \\
\hline Pultencea juniperina & 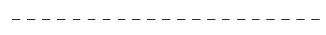 & ------- & $-\ldots---$ & $------1--$ & 111 \\
\hline Tetrarrhena distichophylla & --------------------- & -------- & ------ & $----1-1-1$ & 111 \\
\hline Banksia marginata & $-1-\ldots+\cdots-\cdots,-\ldots--$ & $-\ldots-\ldots$ & $\ldots \ldots-$ & $1--11-m-11$ & 111 \\
\hline Leptospermum glaucescens & $\ldots-1-\ldots \ldots \ldots-n \ldots$ & $\ldots-n-n$ & $-\ldots-\ldots$ & $111-\cdots---1$ & 111 \\
\hline Helichrysum scorpioides & ----------------- & $---\ldots--$ & $-\ldots----$ & $\ldots--1-11$ & 111 \\
\hline Lindsaea linearis & 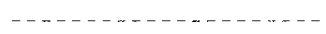 & $---\ldots \ldots-$. & $-\ldots \ldots--$ & $---1-\ldots 1-$ & 111 \\
\hline Selaginella uliginosa & 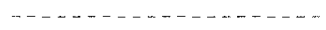 & $-\cdots--\cdots$ & $---\ldots$ & $----1--11$ & 111 \\
\hline Aira caryophyllea & $---\ldots-\ldots-\ldots-\ldots-\ldots-\ldots$ & $-\ldots-\ldots--$ & $\ldots-\ldots$ & $\ldots \ldots-1-$ & 111 \\
\hline Aotus ericoides & 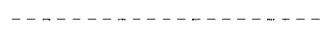 & $---\ldots . n--$ & $--\cdots-$ & $\ldots \ldots \ldots 1$ & 111 \\
\hline Epacris lanuginosa & - & $-\ldots-\ldots$ & $----\cdots$ & $\ldots \ldots-\ldots-$ & 111 \\
\hline Gompholobium huegelii & $-\ldots+\ldots+\ldots$ & ------ & ----- & $-\ldots \ldots--1$ & 111 \\
\hline Gonocarpus teucrioides & 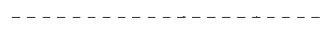 & $-\ldots-\ldots-$ & $\ldots \ldots$ & $---\ldots-1-$ & 111 \\
\hline Lagenifera stipitata & - - - - - - - - - - - - - - & $--\ldots \ldots-$ & $-\ldots \ldots$ & $-\cdots--\cdots-1$ & 111 \\
\hline Leucopogon collinus & $\ldots-\ldots+\ldots-\ldots, \ldots-\ldots$ & $---\ldots--$ & ------ & --------1 & 111 \\
\hline Opercularia varia & 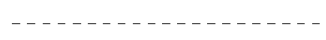 & $---\ldots-$. & $-\cdots---$. & $\ldots-\cdots-1$ & 111 \\
\hline Patersonia fragilis & - - - - - & $\ldots \ldots \ldots-$ & $\ldots \ldots \ldots$ & $\cdots---\cdots---1$ & 111 \\
\hline Pultenaea stricta & 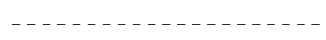 & $-\ldots-\ldots$ & $\ldots-\ldots$ & $\ldots-\ldots-11$ & 111 \\
\hline Deyeuxia quadriseta & $-\ldots-\ldots \ldots \ldots \ldots \ldots-\ldots$ & $\ldots-\ldots$ & $\cdots-\cdots$ & $---\cdots--n-1$ & 111 \\
\hline Sprengelia incarnata & ---------------- & $--\cdots---$ & $-\ldots \ldots-$ & $\ldots \ldots-\ldots 11$ & 1 il \\
\hline Stylidium graminifolium & 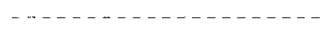 & $-\ldots \ldots$ & $-\ldots-\ldots$ & ------1 & 111 \\
\hline Xanthosia pilosa & ------------------- & $-\ldots \ldots-$ & $-\ldots . .-$ & $-\ldots-\cdots-11$ & 111 \\
\hline Lepidosperma concavum & 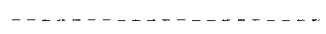 & $\ldots \ldots \ldots-\ldots$ & $\ldots \ldots-\ldots$ & $-\ldots \ldots \ldots-1$ & 111 \\
\hline Schoenus tenuissimus & ------------------- & $----1---$ & $-\ldots-\cdots$ & $-\cdots-1-111$ & 111 \\
\hline Twinspan divisions, & 000000000000000000000 & 00000000 & 1111111 & 1111111111 & \\
\hline two levels & 000000000000000000000 & 11111111 & 0000000 & $111111: 111$ & \\
\hline
\end{tabular}




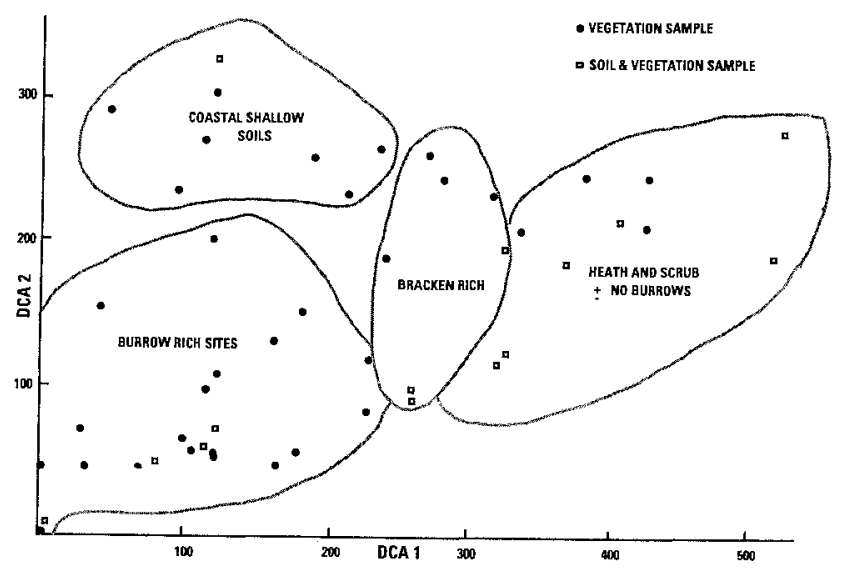

FIG. 2 - Ordination of sites at Pineapples rookery by Detrended Correspondence Analysis of their floristics. Sites where soils samples were taken are indicated. scrub and woodland plots, again with few or no burrows, to sites with old burrows and thence to the lowest DCA score sites with high densities of currently active burrows. Some of the sites having low scores are very rocky, with only very shallow sandy soils. The second axis contrasts these shallow soil sites with the deep sandy sites of high burrow density.

\section{Soil Nutrients}

The complete soils data are given in table 2 . The results of the factor analysis and varimax rotation are given in table 3 . There are highly significant differences for nutrient status between the rookery and non-rookery sites at both locations, with elevated $\mathrm{P}$ and $\mathrm{N}$ concentrations to be found, as expected, on the sites of highest burrow density and/or bird activity $(F=6.06, P<0.05$ for phosphorus and $F=$ 7.07, $\mathrm{P}<0.05$ for nitrogen). There were no sigificant interaction effects found in the ANOVA for $\mathrm{P}$, but there was a highly significant site/burrow occurrence interaction

TABLE 2

Soil chemical analyses from Pineapples and Cape Deslacs rookeries

\begin{tabular}{|c|c|c|c|c|c|c|c|c|c|}
\hline \multirow[t]{2}{*}{ Site* } & \multirow[t]{2}{*}{ Burrows } & \multirow[t]{2}{*}{$\mathrm{pH}$} & \multirow{2}{*}{$\begin{array}{c}\text { Conductivity } \\
\text { TDS } \\
(\mathrm{mS})\end{array}$} & \multicolumn{6}{|c|}{ Elements (ppm) } \\
\hline & & & & $\mathrm{P}$ & K & $\mathrm{N}$ & $\mathrm{Ca}$ & $\mathrm{Mg}$ & $\mathrm{Na}$ \\
\hline 1 & + & 4.6 & 0.40 & 014 & 120 & 3700 & 359 & 271 & 352 \\
\hline 1 & + & 4.4 & 0.33 & 19 & 100 & 3000 & 381 & 171 & 180 \\
\hline 1 & - & 4.2 & 0.24 & 14 & 60 & 400 & 454 & 223 & 168 \\
\hline 1 & + & 4.6 & 0.18 & 45 & 80 & 1400 & 117 & 32 & 45 \\
\hline 1 & + & 5.1 & 0.14 & 12 & 50 & 3000 & 201 & 101 & 130 \\
\hline 1 & + & 4.5 & 0.23 & 140 & 80 & 3000 & 186 & 121 & 152 \\
\hline 1 & + & 6.1 & 0.43 & 14 & 320 & 3400 & 311 & 394 & 518 \\
\hline 1 & + & 5.0 & 0.94 & 53 & 310 & 4000 & 685 & 311 & 713 \\
\hline 1 & + & 4.6 & 0.37 & 130 & 90 & 4500 & 274 & 91 & 112 \\
\hline 1 & + & 4.7 & 0.38 & 28 & 90 & 1600 & 195 & 216 & 353 \\
\hline 1 & - & 4.9 & 0.22 & 9 & 120 & 700 & 107 & 128 & 210 \\
\hline 1 & - & 5.2 & 0.24 & 9 & 90 & 1100 & 242 & 465 & 287 \\
\hline 1 & - & 4.9 & 0.16 & 5 & 80 & 800 & 81 & 210 & 170 \\
\hline 1 & - & 4.9 & 0.09 & 6 & 50 & 1600 & 337 & 387 & 139 \\
\hline 2 & + & 5.3 & 0.15 & 17 & 80 & 800 & 216 & 120 & 198 \\
\hline 2 & + & 5.3 & 0.10 & 24 & 50 & 200 & 41 & 28 & 82 \\
\hline 2 & + & 5.3 & 0.12 & 16 & 60 & 400 & 95 & 68 & 111 \\
\hline 2 & + & 5.7 & 0.08 & 29 & 60 & 300 & 109 & 89 & 81 \\
\hline 2 & - & 6.1 & 0.16 & 5 & 350 & 800 & 230 & 503 & 388 \\
\hline 2 & - & 5.6 & 0.37 & 12 & 310 & 1100 & 628 & 223 & 352 \\
\hline 2 & + & 5.3 & 0.16 & 9 & 30 & 200 & 56 & 58 & 87 \\
\hline 2 & + & 5.5 & 0.25 & 6 & 140 & 700 & 158 & 159 & 314 \\
\hline 2 & + & 5.4 & 0.15 & 13 & 50 & 400 & 150 & 125 & 106 \\
\hline 2 & + & 6.1 & 0.04 & 1 & 40 & 200 & 65 & 64 & 46 \\
\hline 2 & + & 5.7 & 0.10 & 56 & 60 & 600 & 201 & 64 & 96 \\
\hline 2 & + & 5.7 & 0.22 & 48 & 70 & 900 & 333 & 165 & 184 \\
\hline 2 & - & 6.0 & 0.26 & 5 & 220 & 700 & 471 & 310 & 527 \\
\hline 2 & - & 6.1 & 0.22 & 6 & 300 & 900 & 438 & 384 & 410 \\
\hline 2 & - & 6.2 & 0.28 & 8 & 380 & 1800 & 211 & 495 & 749 \\
\hline 2 & - & 6.7 & 0.55 & 9 & 380 & 1400 & 681 & 365 & 710 \\
\hline 2 & - & 6.1 & 0.23 & 7 & 130 & 500 & 196 & 121 & 247 \\
\hline
\end{tabular}

${ }^{*} 1$ - Pineapple; 2 - Cape Deslacs. 
TABLE 3

Varimax rotated factor matrix of soil variables and ordination scores*

\begin{tabular}{lcr}
\hline Variable & R.F.1 & R.F.2 \\
\hline $\mathrm{pH}$ & $0.523 \dagger$ & -0.058 \\
$\mathrm{TDS}$ & 0.790 & 0.435 \\
$\log \mathrm{P}$ & -0.102 & 0.905 \\
$\log \mathrm{K}$ & 0.789 & 0.356 \\
$\log \mathrm{N}$ & 0.316 & 0.738 \\
$\log \mathrm{Ca}$ & 0.644 & 0.226 \\
$\log \mathrm{Mg}$ & 0.770 & -0.459 \\
$\log \mathrm{Na}$ & 0.940 & -0.064 \\
DCA1 & -0.260 & -0.920 \\
DCA2 & 0.457 & -0.583 \\
\hline
\end{tabular}

* Data from the Pineapples rookery

+ Bold type indicates values greater than 0.5 irrespective of sign.

for $N(F=32.36, P<0.001)$, reflecting the high variability of the soils at the Pineapples, where the differences between the high $\mathrm{N}$ concentrations in the bare areas and those of the heaths are very much more pronounced than in equivalent areas at Cape Deslacs, where no clear differences between burrow and non-burrow vegetation were apparent. That this trend is reflected in the vegetation composition is borne out clearly in the varimax rotation scores (table 3 ). Interestingly, the first factor reflects a saline influence, with $\mathrm{Na}, \mathrm{TDS}, \mathrm{K}, \mathrm{Mg}$ and $\mathrm{Ca}$ being most highly weighted. Neither DCA axis is aligned strongly with this factor, suggesting that the major compositional trends in the vegetation are related to factors other than direct salinity effects, at least at this site, over the range of concentrations recorded. The direction of floristic variation may also be oblique to the direction of the saliniry gradient. However, the second factor axis shows a very strong relationship between DCA 1, the major vegetation gradient, and a combination of $\mathrm{N}$ and $\mathrm{P}$ concentration, i.e. soil variables which relate directly to nutrient status. In fact, the second DCA axis grades the sites in order of soil depth, amount of organic matter incorporated in the soil (chiefly grass) and the density of burrows in combination. Thus it appears that, given the halophytic natures of the species involved, the succession of vegetation in the area is biogenically determined and does not reflect directly the usual underlying physical parameters, such as rock type, drainage or onshore effects of salinity. There is some evidence in the field of the residual vegetation which is so determined; e.g. the TWINSPAN analysis separates the group of Melaleuca spp. characteristic of poorly drained sites.

\section{DISCUSSION}

The vegetation of shearwater rookery sites is subject to stresses additional to those offered elsewhere in coastal environments. Surface-nesting birds, such as albatross and cormorants, give rise to considerable trampling and, of course, high levels of guano deposition. Guano is rich in phosphorus and nitrogen to the extent that it can be phytotoxic, especially in regions subject to drought (Gillham 1960). The vegetation in nesting areas of burrowing birds is prone to disturbances similar to those caused by surfacenesting birds but the burrows are usually (and necessarily!) located in areas of deeper soil development. Here, the use of sites is dependent on depth and friability of soils and on the likelihood of the burrow sites remaining free from inundation by water or from structural collapse, for the duration of use.

The shearwaters alter the habitat in the same way as surface-nesting birds by guano deposition and by trampling (especially in marshalling areas), but they also redistribute and incorporate surface organic matter into the soil, and they increase the degree of aeration of the soil volume.

The mechanical damage caused by the birds can lead to soil compaction ("hard ground") and also to pedestalisation of Poa tussocks. The bare areas are eventually colonised by Tetragonia and Carpobrotus after abandonment by the birds or through leaching of excess nutrients within the soil profile. These bare areas are often initially very nutrient rich and, in areas where a source of seed is available, they will support a flush of growth of exotic winter annuals such as Avena, Hordeum and Bromus spp., together with thistles and other Asteraceae. Such species are able to utilise the nutrients during the wet months and have set seed and died back by summer, when the likelihood of drought and nutrient toxicity becomes a problem for the less aggressive perennial native species.

Gillham (1965) considered that fire constituted a major cause of rookery depletion and cited altered levels of soil organic content in previously occupied sites after fire as evidence of this effect. Similar areas of "hard ground" were observed in parts of the present study areas and also, more extensively, in the commercial rookery areas on Great Dog Island (MJB, pers. obs.). The intensity and frequency of burning are known to be responsible for organic matter depletion in other Tasmanian ecosystems (e.g. Bowman et al. 1986). Fires may also kill the birds directly or cause abandonment of the sites, and may subsequently result in collapse of the system, since it is the birds which are responsible for the organic matter incorporation in the first place.

Thus, the succession in the rookery sites studied is strongly under the control of the birds themselves, but there is a pronounced effect also from human sources especially via fire or introduced weeds, as well as direct exploitation and physical destruction of burrows.

\section{ACKNOWLEDGEMENTS}

We wish to acknowledge the asistance of D. Brown and the support of the Toyota Foundation in the preparation of this work.

\section{REFERENCES}

BOWden, A.R. \& KIRKPATRICK, J.B., 1974: The vegetation of the Rheban Spit, Tasmania. Pap. Proc. R. Soc. Tasm. 108: 199210.

Bowman, D.M.J.S., Maclean, A.R. \& Crowden, R.K., 1986: Vegetation-soil relations in the lowlands of south-west Tasmania. Aust. J. Ecol. 11: 141-153.

Brown, M.J., 1980: The vegetation of the Mt. Cameron West Aboriginal Site. Pap. Proc. R. Soc. Tasm. 114: 21-34.

Brown, M.J. \& PODGER, F.D., 1982: Floristics and fire regimes of a vegetation sequence from sedgeland-heath to rainforest at 
Bathurst Harbour, Tasmania. Aust. J. Bot. 30: 659-676.

Buchanan, A., MCGeary-Brown, A. \& Orchard, A.E., 1989: $A$ CENSUS OF THE VASCULAR PLANTS OF TASMANIA. Occ. Publ. Tasm. Herb. 2.

Chladil, M.A. \& KirkPaTrick, J.B., 1989: A transect study of the sand dune vegetation at Bakers Beach, Tasmania. Pap. Proc. R. Soc. Tasm. 123: 247-256.

Grllham, M.E., 1958: Chappell Island, Eastern Bass Strait. Preliminary report to the Tasmanian Fauna Board on vegetation and suggestions regarding future management. Unpubl. rep. NPWS, Tasm.

Glliham, M.E., 1960: Destruction of indiginous heath vegetation in Victorian sea bird colonies. Aust. J. Bot. 8: 277-317.

GIllHam, M.E., 1961: Plants and sea-birds of granite islands of South-East Victoria. Proc. R. Soc. Vict. 74: 21-36.

Gillham, M.E., 1965: The Fisher Island Field Station IV: Vegetation additions and changes. Pap. Proc. R. Soc. Tasm. 99: 71-80.

HIL, M.O., 1979a: DECORANA-a FORTRAN program for detrended correspondence analysis and reciprocal averaging. Ecology \& Systematics Dep., Cornell Univ., New York.

HiLl, M.O., 1979b: TWINSPAN-a FORTRAN program for arranging multivariate data in an ordered two-way table by classification of individuals and attributes. Ecology \& Systematics Dep., Cornell Univ., New York.
JACKSON, W.D., 1968: Fire, air, earth and water - an elemental ecology of Tasmania. Proc. Ecol. Soc. Aust. 3: 9-16.

KIRKPATRICK, J.B., 1977: THE DISAPPEARING HEATH. Tasmanian Conservation Trust Inc., Hobart: 95 pp.

NaArding, J.A., 1981: Study of the short-tailed shearwater Puffinus tenuirostris in Tasmania. Tech. Rep. Wildl. Div. 81/3. NPWS, Tasm.

Serventr, D.L., 1958: Recent studies on the Tasmanian muttonbird. Aust. Mus. Mag. 12: 327-332.

Servinty, D.L., 1967: Aspects of the population ecology of the short-tailed shearwater Puffinus tenuirostris. Proc. XIV Int. Ornithol. Congr.: 165-190.

Serventy, V., 1968: WILDLIFE OF AUSTRALIA. Nelson, Melbourne.

SKIRA, I.J., 1990: Human exploitation of the short-tailed shearwater (Puffinus tenuirostris). Pap. Proc. R. Soc. Tasm. 124: 77-90.

Skira, I.J., Wapstra, J.E., Towney, G.N. \& NaAroing, J.A., 1986: Conservation of the short-tailed shearwater Puffinus tenuirostris in Tasmania, Australia. Biol. Cons. 3: 225-236.

(accepted 18 September 1992) 